Extrême-Orient Extrême-Occident

\section{Extrême-Orient Extrême-Occident}

$32 \mid 2010$

Faux et falsification en Chine, au Japon et au Viêt

Nam

\title{
Vraies et fausses vierges au Viêt Nam. La falsification corporelle en question
}

Real and Fake Virgins in Vietnam - Questioning Body Falsification

\section{Trọng Hiếu Đinh}

\section{(2) OpenEdition}

\section{Journals}

Édition électronique

URL : http://journals.openedition.org/extremeorient/115

DOI : 10.4000/extremeorient. 115

ISSN : 2108-7105

Éditeur

Presses universitaires de Vincennes

Édition imprimée

Date de publication : 1 octobre 2010

Pagination : 163-191

ISBN : 978-2-84292-263-4

ISSN : 0754-5010

Référence électronique

Trọng Hiếu Đinh, « Vraies et fausses vierges au Viêt Nam. La falsification corporelle en question », Extrême-Orient Extrême-Occident [En ligne], 32 | 2010, mis en ligne le 01 octobre 2013, consulté le 01 mai 2019. URL : http://journals.openedition.org/extremeorient/115;DOI : 10.4000/extremeorient.115 


\title{
Vraies et fausses vierges au Viêt Nam La falsification corporelle en question
}

\author{
Đinh Trọng Hiêu
}

\section{Introduction}

$\mathrm{Au}$ Viêt Nam, il semble que la virginité féminine et sa falsification n'ont jamais cessé de coexister. Si cette virginité continue à être considérée comme l'une des «vertus féminines majeures » (sinon la première), les pratiques pour la simuler ne sont plus cachées, elles sont parfois même revendiquées. Cela nous a semblé représenter les deux facettes d'une réalité socio-culturelle, où le vrai et le faux restent intimement mélangés, l'un et l'autre n'existant peutêtre pas sans leur contrepartie. Néanmoins, cette falsification touche le corps humain qui devient l'objet de manipulations plus ou moins frauduleuses. La difficulté d'investigation n'en est que plus exacerbée. Sur cette question, on ne saurait dire que la documentation abonde, ancienne ou récente, aussi bien au Viêt Nam qu'en Chine. Il n'y a aucun témoignage sur cet aspect dans les écrits concernant le Viêt Nam, qu'ils soient historiques ou littéraires, hormis une allusion dans le roman versifié Kiêu. Même pour la Chine ancienne, l'ouvrage de Robert Van Gulik ${ }^{1}$, pourtant bien documenté, ne contient que peu de pages relatives à la virginité féminine; rien n'y a été trouvé sur les simulacres pour devenir fausses vierges.

Pourquoi, dès lors, nous lancer dans une telle étude?

Son intérêt est multiple. D'abord, la problématique de la distinction entre le vrai et le faux, appliquée au corps humain: peut-on effectuer une «falsification corporelle», ne se limite-t-elle qu'à un point de détail ? Quelles en sont les implications - physiques, émotionnelles, morales, sinon pénales - dans un pays où le «faux », l'imitation ou la falsification ne sont pas nécessairement réprouvés? En second lieu, puisqu'il s'agit d'actes pratiqués d'humains à humains, on

1. Robert Van Gulik, Sexual Life in Ancient China, 1961. Traduction en français par Louis Evrard, La Vie sexuelle dans la Chine ancienne. Paris, Gallimard, «Tel», 1971. 
peut interroger ces divers acteurs: on essaiera de cerner non seulement leurs motivations, mais encore leur vécu, leurs représentations. Cette dimension nous paraît importante, elle fait partie de la démarche anthropologique, qui sera envisagée, ici, d'une manière holistique : ainsi, l'individu sera abordé dans son ensemble, même si seulement une infime partie de sa personne physique est concernée. Il nous appartiendra de nous interroger encore sur cet aspect particulier du domaine de la «sexualité»: pourquoi ce «succès » à la fois des simulacres de la virginité et de l'hyménoplastie ${ }^{2}$, à l'heure actuelle, et dans des sociétés non-européennes? Que sous-entend un tel recours technique: une évolution des mœurs féminines, plus libérées au Viêt Nam «socialiste», ou, au contraire, une expression du machisme dans cette société, ce qui a pour conséquence de contraindre la femme à de telles pratiques? Est-ce simplement un aspect ludique de la sexualité masculine, une recherche de l'érotisme? Y voit-on une possibilité de «reconstruire» la personnalité féminine ? N'y a-t-il pas aussi d'autres enjeux à l'heure de l'économie de marché ? En dernier lieu, est-ce un phénomène localisé au seul Viêt Nam, n'a-t-il pas vocation à déborder ces limites ? On entrevoit d'ores et déjà la multiplicité des implications d'un tel sujet. Aussi nécessite-t-il une étude étape par étape.

\section{Le vrai et le faux dans le contexte socio-culturel}

Le couple vrai/faux, se dit thật 實/giả 假. Ce sont des termes en usage dans la vie courante, au moins depuis le XVII ${ }^{\mathrm{e}}$ siècle $^{3}$, bien que d'origine chinoise. La combinaison de ces deux termes n'implique aucune hiérarchisation: on peut dire invariablement thật/giả, ou giả/thật: «thật giả (ou giả thật) khó phân» («il est difficile de distinguer le vrai du faux»). Thât, avec son doublon thưc, n'est pas usuellement employé, car si quelque chose est réel, vrai, on le désigne par son appellation habituelle, sauf quand on veut insister sur cette dimension du vrai, ainsi nói thật («parler vrai»), húa thật («vraie promesse»). Giả peut être à la fois, comme maints termes du vietnamien, adjectif, verbe, ou adverbe, selon sa place: placé après un substantif, il est adjectif et signifie «faux» sans connotation péjorative (ou laudative). «Faire du faux», «falsifier», n'est pas

2. L'hyménoplastie, est-il besoin de le rappeler, requiert une habileté particulière du chirurgien pour une totale réussite, voir infra.

3. Giả et thật sont attestés notamment dans le Dictionnaire d'Alexandre de Rhodes, respectivement pour le premier à la page 272 (falsus), et à la page 751 pour le second, dans la graphie thặt (veritas, verus). Cf. Alexandre de Rhodes, Dictionarium annamiticum, lusitanum et latinum, Rome, 1651.

4. Ancienne graphie: dả. 
Vraies et fausses vierges au Viêt Nam. La falsification corporelle en question

toujours une pratique condamnable au Viêt Nam, et, très couramment, on peut trouver nombre d'exemples de cet usage du faux.

L'emploi des billets «monnaie du Monde obscur» est courant dans toute l'Asie sinisée : ce sont de faux billets destinés à être brûlés pour les besoins des disparus ${ }^{5}$. Au Viêt Nam ils sont parfois reproduits, à l'instar de vrais billets, avec l'effigie du «fondateur de l'État socialiste » en personne, en de très grosses coupures. Ainsi, au moins deux mondes existent, monde obscur des disparus, et monde lumineux des vivants. Les vivants achètent avec du vrai argent non seulement de la «fausse monnaie», de faux lingots, de faux feuillets d'or, d'argent, mais encore toute une panoplie de faux objets destinés à être brûlés, comme objets votifs : faux chevaux, fausses mobylettes Honda, fausses voitures, faux buildings, voire fausses concubines parées des plus beaux atours. Le feu fait passer ces «faux» dans l'autre monde, où ils vont servir. Ces faux n'ont pas un statut d'infériorité, teinté de mépris, au contraire. À Hanoi, l'une des petites rues de la «vieille ville », Phố Hàng Mã («Rue des ex-votos»), toujours très achalandée, reste une devanture pour les inventions de «faux» dont sont capables les artisans vietnamiens, désormais regroupés en un «village de métiers» (làngnghề) tout à fait prospère. Des objets réels, mais de facture fragile, sont dénommés, par un processus d'inversion, des «objets [analogues aux] ex-votos » (đô mã).

On assiste à la même inversion des valeurs dans une anecdote des années 1980, à l'allure de parabole ${ }^{6}$. En ces temps de pénurie alimentaire, un citadin, désespéré de voir sa famille acculée à une misère sans issue, décide de recourir au suicide collectif. Il s'en va au marché acheter de la mort-aux-rats qu'il répand en secret dans le potage et le sert à tous ses proches. Après une longue nuit de sommeil, tout le monde se réveille cependant, vivant. L'homme comprend alors qu'au marché, en période d'indigence de produits, on lui a vendu une mort-auxrats trafiquée: ce (faux) poison ne s'avère pas létal. Fou de joie, notre homme retourne au marché acheter du glutamate ${ }^{7}$ pour le mettre dans un autre potage servi en grande pompe, en signe de fête. Malheureusement, le lendemain, aucun membre de la famille ne s'est réveillé, le repas festif s'est révélé fatal à tous, car, cette fois, c'est le glutamate qui avait été trafiqué. Ainsi, un faux poison

5. Cf. François Thierry, Amulettes de Chine et du Viêt-Nam, Paris, Le Léopard d'or, 1987, p. 23.

6. Cette anecdote fut collectée lors de notre mission CNRS-Université Paris Diderot-Paris 7, fin avril-juillet 1982. De tels textes, partie intégrante de la «littérature orale», circulaient, à l'instar des samizdats en Union Soviétique, sans qu'aucune censure officielle ne puisse les arrêter.

7. Le glutamate est un exhausteur de goût, très prisé dans la cuisine asiatique, notamment quand on ne dispose pas de bouillon de viande pour relever la plupart des mets. 
est salvateur alors qu'un additif alimentaire, quand il est trafiqué, peut s'avérer mortel.

D'autres imitations dans le domaine culinaire sont moins dangereuses, et restent très prisées. L'imitation tend à contourner des interdits alimentaires, à produire des plats de substitution en cas de pénurie, mais surtout est destinée à démontrer la maîtrise du «faux» dans l'art d'accommoder des mets. Le terme giả, placé devant une matière alimentaire, signifie «imitation de». Le giả ba ba est un ragoût de porc (viande commune) qui tend à imiter la chair de la tortue d'eau (plat plus rare et chair plus estimée), ou le giảcâyy, plat très sophistiqué de viande de porc, pour imiter du chien ${ }^{8}$. L' «imitation culinaire» ne se cantonne pas à la reproduction gustative d'un plat, d'un mets, elle a l'ambition d'en restituer aussi l'aspect. Dans la confection des plats végétariens, il existe une longue tradition d'imitation de l'apparence des mets de viande, ainsi, lors des banquets dans les temples bouddhiques, sert-on aux convives du «faux »: faux «jambon» avec couenne, gras, chair rosée, faux travers de porc avec des bouts de côtes, etc., le tout confectionné avec de la pâte de soja comme principal ingrédient. L'art de l'imitation tend à la perfection dans la fabrication des faux objets : fausses fleurs, faux animaux sculptés à partir de simples fruits ou légumes lors des concours d'art ménager. C'est cette volonté de restituer (voire dépasser) la réalité par le faux qui est remarquable et qui la distingue de la simple utilisation de fausses fleurs dans la décoration, dans d'autres sociétés.

Appliqués au corps ou à ses composantes, les artefacts ne semblent étonner plus personne. Point d'opposition de valeur entre ce qui existe naturellement et ce qui existe artificiellement. Le faux remplace le vrai quand celui-ci n'existe plus. Dans un pays qui a longtemps connu la guerre, la plupart des interventions réparatrices sont ressenties comme une nécessité, souvent bienfaisante. Il n'y a pas lieu de déprécier une prothèse, car la «fausse » partie du corps constitue une aide précieuse pour l'individu et contribue à améliorer son existence : chân giả (fausse jambe, prothèse du membre inférieur), răng giả (fausse dent, prothèse dentaire), mắt giả (faux œil, prothèse oculaire), etc. En raison de l'attrait des modèles occidentaux, les faux ou artefacts corporels se sont multipliés, parmi lesquels les «faux seins» (vú giả ${ }^{9}$ font fureur: des jeunes filles viennent les choisir et les essayer en public, souvent en compagnie de leur petit ami. Avec

8. Le giả cây, initialement, était un plat utilisant la viande de porc pour imiter celle de la civette (cây, ou câyy hương, civette porte-musc). Actuellement il sert à désigner le plat imitant la viande de chien. Le chien, animal domestique et disponible à tout moment au Viêt Nam, a une chair échauffante, recommandée seulement à partir du $22^{\mathrm{e}}$ jour du mois lunaire. D'où son imitation dans la cuisine domestique et/ou gastronomique.

9. Morceaux de mousse à glisser dans les soutiens-gorge, ou ces mêmes «lingeries » déjà rembourrées, d'importation chinoise, prêtes à l'emploi. 
l'essor de l'esthétique et de la chirurgie réparatrice, alors que le niveau de vie s'accroît en milieu urbain, est apparue toute une panoplie de parties corporelles, généralement féminines, affublées du terme giả : cầm giả («faux menton»), lông mày giả («faux sourcils»), lông mi giả («faux cils»), mũi giả («faux nez»), vú giả («faux seins», prothèse mammaire et non simple rembourrage externe, aux mêmes effets, nommés pareillement), etc. Ce «faux» n'épargne pas non plus la gent masculine. Autrefois, dans les harems de la dernière dynastie impériale, des eunuques défloraient les odalisques, à l'aide d'un faux pénis en ivoire, dans le but d'épargner au monarque une fatigue supplémentaire, lors de ses relations sexuelles. Actuellement, certains hommes fortunés ont recours à la chirurgie réparatrice pour allonger ou renforcer leur membre viril, mais cette pratique, comme la précédente, n'est pas encore du domaine de la vie courante et ne suscite guère de réprobation.

L'usage du «faux» faisait et fait encore partie intégrante de l' ' art militaire», non seulement par l'utilisation des «leurres», du camouflage, mais surtout en recourant aux feintes, simulacres, ou «manœuvres de diversion» (nghibinh 疑兵). Ces constantes se retrouvent au cours des siècles, face aux différents ennemis, elles jalonnent l'histoire militaire du Viêt Nam, du XIII' au Xx siècle. Cette «culture du faux » s'épanouit même dans des situations où il n'y a aucune nécessité impérieuse, notamment dans le domaine de la créativité artistique. Ainsi, la conception muséographique en vigueur ne fait pas de distinction entre une réfection et l'œuvre originelle: une vieille sculpture sur bois originelle est tout simplement jetée, remplacée par une sculpture analogue, moderne, plus ou moins habile ${ }^{10}$. Certains artistes, connus, effectuent eux-mêmes des copies de leurs œuvres «disparues»; ces copies sont actuellement exposées au musée des Beaux Arts, sans qu'il y ait une quelconque spécification de leur état. Les musées disposent eux-mêmes d'un service de reproduction d'œuvres, les copies sont exposées en lieu et place des œuvres originales ${ }^{11}$, toujours sans spécification. C'est la famille même d'autres artistes, en principe garante de l'authenticité des œuvres, qui procède elle aussi à des copies qu'elle tentera de faire authentifier par la suite. Il y a là de quoi tromper, non seulement de simples visiteurs de musées, mais encore des «experts», pourtant avertis ${ }^{12}$.

10. Il ne s'agit pas, ici, d'une discussion sur le degré d'habileté (plus ou moins grand) de la réfection, mais de la notion de l'«authenticité». Sinon, nous avons assisté à des démonstrations de virtuosité de la part de certains jeunes artisans qui semblent même dépasser les anciens maîtres dans l'exécution des réfections d'œuvres fort compliquées.

11. Car ces œuvres appartiennent à des collections privées.

12. On peut relever, dans les ouvrages sur l'art du Viêt Nam, maintes reproductions de copies de tableaux, sans aucune mention de leur état. 
Ainsi, les «faux » ne suscitent pas généralement de réprobation. Deux cas semblent faire exception: en premier lieu, les mots ou expressions forgées avec le verbe mạo 冒, (mạo chũ ký, «contrefaire une signature», mạodanh, « usurper le nom [de quelqu'un] », giả mạo giây tò, «contrefaire des papiers »), autant de pratiques stigmatisées par la morale et réprimées par la loi, désignées essentiellement avec l'emploi de mạo («contrefaire»). En deuxième lieu, dans l'expression giả đạođúc (ou son inverse: đạo đúc giả), qui signifie «faire l'hypocrite», «être hypocrite», la connotation péjorative est due à la présence des termes qui signifient «vertus» (đạođúc 道德), et dont la qualité ne peut souffrir de contrefaçon, et non à la contrefaçon elle-même.

Qu'en est-il quand, au Viêt Nam, on falsifie la virginité?

\section{Virginité et défloration}

En vietnamien, le vagin s'appelle cưa mình («porte du corps » ou «porte du moi intime»). Âm đạo 陰道 (《conduit, chemin yin») est une autre appellation d'après la terminologie savante, chinoise ${ }^{13}$. Vers la fin de l'adolescence, une mince membrane, plus ou moins plissée et fragile, appelée «hymen», obstrue cette «porte», à environ un centimètre du bord. Cette membrane est parcourue de vaisseaux sanguins, sa déchirure provoque habituellement une petite hémorragie, mais celle-ci n'est pas systématique: elle survient lors du premier rapport sexuel; l' «hymen», c'est-à-dire le «mariage» est, dès lors, consommé. Mais cette rupture peut être accidentelle, lors d'une chute, d'un grand et brusque écart. L'usage moderne du tampon hygiénique peut avoir les mêmes résultats ${ }^{14}$. Ce sont là les traits principaux de la constitution féminine et universelle : les caractéristiques du corps de la femme vietnamienne ne dérogent pas à ces généralités. En revanche la terminologie relative à la virginité est

13. Vulgairement, l'appellation vietnamienne est : lỗ lồn («trou de la vulve»).

14. L'hymen ne ferme pas complètement le conduit vaginal, il $\mathrm{y}$ a un orifice par où s'écoule le sang des menstrues. Cet orifice peut avoir des formes différentes : ronde, ovale, en arc de cercle, folliculaire, réniforme... À titre indicatif, une «enquête» du journal en ligne VnExpress auprès de 9600 Vietnamiennes donne ces résultats : $54 \%$ ont une hémorragie lors des premiers rapports, $17 \%$ ne savent pas, $29 \%$ disent qu'il n'y a aucune signe (cité d'après Talawas, 08/2005, http://www.talawas.org/talaDB/showFile. php ? res $=5703$ $\& \mathrm{rb}=0508$ ). Dans le «courrier du cœur» de la presse féminine et locale, on trouve maintes questions (angoissantes) concernant l'absence d'hémorragie lors du premier rapport sexuel. Mais on peut aussi lire, sous la plume de Buffon, comment une jeune-fille qui a eu des commerces sexuels avant la puberté peut présenter tous les «signes» de la virginité une fois l'âge de la puberté atteint, voir Leclerc, comte de Buffon, Histoire naturelle, générale et particulière, avec la description du cabinet du Roy, Paris, 1749, t. II, p. 492-502. 
singulière. L'hymen est dénommé màng trinh. Màng désigne quelque chose d'une extrême minceur, d'une grande fragilité, l'appellation suggère par ellemême un traitement fort délicat à son égard, pour sa préservation. La virginité n'est pas un concept abstrait, elle fait référence directement à cette membrane. Une jeune fille est, soit encore vierge, et on dit qu'elle dispose «encore de son hymen » (còn trinh), soit déflorée, et on dit alors qu'elle a "perdu son hymen» (mât trinh). L'acte de défloration utilise un verbe qui exprime la brutalité: phá trinh («détruire l'hymen»), ce qui n'implique pas forcément une réelle violence à l'égard de la jeune fille, lors de cette «destruction», sauf dans le cas du viol. Trinh 貞, utilisé seul, suffit à désigner l'état de «virginité»; jadis, le mot était souvent couplé avec tiêt 節, ( droiture, fidélité»), pour désigner «virginité et chasteté». Trinh tiêt 貞節, l'expression se rapporte à une jeune fille «vierge» avant le mariage et «fidèle» par la suite à son mari. C'étaient, dans le Viêt Nam classique, les vertus cardinales d'une femme.

L'éducation sexuelle n'existe pas au Viêt Nam, surtout en milieu rural. Cependant, au sein de la famille, quelle qu'elle soit, la mère supplée à cette carence en prodiguant des conseils sur la préservation de l'hymen, toujours considéré comme le nécessaire témoin de la virginité. Ces conseils, avec les soins au moment des menstrues, constituent l'essentiel de ce que doit connaître une jeune sur son corps. Qu'une adolescente se tienne, innocemment, les jambes écartées, sa mère la gronde en lui rappelant de «faire attention, quand on s'assoit» (ngồi có ý tú), d'éviter d'avoir «les cuisses largement écartées », «jusqu'à écarter son sexe» (ngồi tô hô ra, ngồi dạng tè he), ou en lui disant, brutalement, que c'est une «posture de dévergondée » (ngồi nhu' con đĩ). On lui apprend à s'accroupir en fermant ses cuisses et à les tenir rabattues d'un même côté, dans une posture typique, souvent immortalisée par les artistes vietnamiens, peintres et sculpteurs ${ }^{15}$. C'était la seule manière féminine de se tenir tant qu'on s'asseyait sur une large surface plate, à même le sol, sur une natte ou sur un bat-flanc. Ce n'est qu'avec l'émergence des chaises, du mobilier moderne, que sont apparues quelques variantes dans ce maintien. D'autres recommandations maternelles visent à aiguiser l'attention des filles: il ne faut pas grimper aux arbres, il faut prendre garde à ce qu'aucun bâton, qu'aucune fourche ou objet pointu n'inflige des blessures irrémédiables à cette partie intime. On leur apprend à monter à bicyclette avec mille précautions, on choisit le modèle de cycle

15. Quelques exemples: Nam Sơn, «Portrait de paysanne assise» (1931), Vũ Cao Đàm, «Jeune fille assise» (circa 1939-1944, sculpture), Tô Ngọc Vân, «Jeunes femmes avec bambin» (1944). 
«pour dame ${ }^{16}$, sans monture latérale haute, ce qui n'est pas sans rappeler une attention analogue en Occident, jadis, aux temps où les dames montaient encore à cheval «en amazone». La jeune fille évite, de même, tout sport violent, ou tout mouvement à risques. Dans leurs relations avec les jeunes gens, le flirt est privilégié, superficiel, sans pénétration; la jeune fille développe toute une tactique pour plaire à son partenaire et, en même temps, préserver sa virginité, uniquement vaginale. La mère rappelle, à ce propos, qu'il y a toujours un risque, car on peut «rester sage pendant trois ans, et devenir sotte ne serait-ce qu'une heure » (khôn ba năm, dại một giơ)), un seul instant d'inattention et la jeune fille va perdre ce qu'elle a de plus précieux!

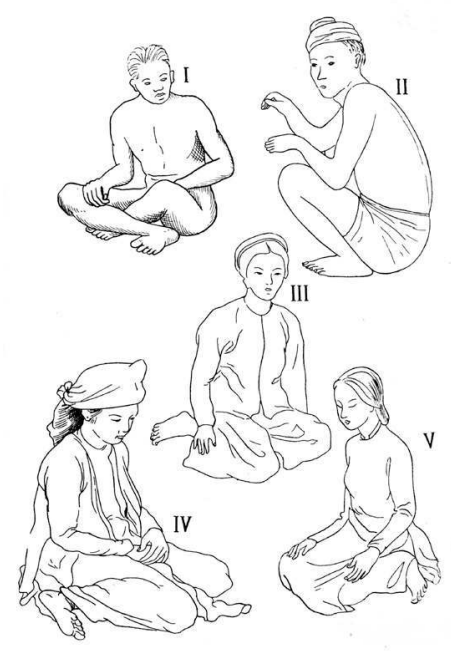

Fig. 1. Postures accroupies chez les Vietnamiens, d'après Đỗ Xuân Hợp ${ }^{17}$ : I. Position dite «en tailleur», homme, tous milieux. II. Position accroupie sur les talons, homme (ou femme âgée), milieu paysan. III. Position accroupie d'une femme, milieu urbain. IV.Position accroupie d'une femme, milieu paysan. V. Position accroupie d'une jeune fille, milieu urbain. (Légendes par l'auteur de l'article.)

16. C'est le même modèle qu'en Occident, mais infiniment plus cher que le modèle «pour homme».

17. Dr. Đỗ Xuân Hợp (Chef des travaux d'anatomie à la faculté de médecine), «Adaptation des os du membre inférieur des Annamites à la marche et à la position accroupie», Bulletins et Travaux de l'Institut Indochinois pour l'Étude de l'homme, Hanoi, 1942, fasc. 1-2, p. 125-133. 
Cette vigilance doit être permanente même si elle perturbe passablement les relations entre jeunes: toujours rester sur le qui-vive quand une jeune fille est en tête à tête avec un garçon, pour garder son «unique bien»! Car cette perte, en dehors des liens du mariage, reste, la plupart du temps, un cauchemar. Ainsi, à un âge déjà mûr, Kim Lefèvre évoque, dans son livre autobiographique, cette frayeur d'adolescente:

J'étais atterrée. La peur de perdre ma virginité me rendait malade. Je faisais des cauchemars où un homme grand et âgé, muni d'un sabre, me chassait à coups de pied en criant: «Elle a perdu sa virginité !» La foule massée autour de moi me couvrait de crachats. J'essayais de me défendre mais aucun son ne sortait de ma bouche ${ }^{18}$.

Quels sont les signes extérieurs de la défloration? La «membrane de la virginité» (l'hymen, màng trinh) n'étant pas visible, sauf par auscultation médicale, ni ressentie lors des relations sexuelles, on se fondait sur un signe quasi «universel» survenu lors de sa rupture: une hémorragie plus ou moins importante. Le drap (ou une pièce de tissu) taché de sang et montré le lendemain des noces avait valeur de test. Au Viêt Nam, cette pratique a perduré jusqu'au milieu du $\mathrm{Xx}^{\mathrm{e}}$ siècle environ. Elle fut abandonnée sous l'impulsion du «Tự lực Văn Đoàn [Groupe littéraire autonome, 1934]». Avant tout littéraire, ce mouvement moderniste ne se détournait pas des problèmes de «modernisation des mœurs », il y contribua même avec force ${ }^{19}$. Nul ne peut oublier dans le roman Đoạn Tuyệt [La rupture, 1935] de son chef de file, l'écrivain Nhất Linh, l'épisode qui scelle la rupture avec des mœurs désormais jugées archaïques: Loan, l'hérö̈ne, jeune fille émancipée, manifeste son mépris quand elle surprend son futur mari en train d'étendre un linge sur le lit, la nuit de leurs noces, «Rien n'est plus précieux que la pureté de l'âme», se dit-elle ${ }^{20}$. Néanmoins, ce «test» de l'hémorragie est resté ancré comme une preuve irréfragable de la virginité, croyance qui confine parfois à l'irrationnel. En témoigne cette «expertise» traditionnelle et macabre, rapportée en 1943 par un médecin dans la rubrique «Morts par surmenage sexuel»: «Pour reconnaître si une victime est encore vierge, on se

18. Kim Lefèvre, Métisse blanche, Paris, Bernard Barrault, 1989, p. 111.

19. On lira avec intérêt les débats, quelque peu académiques, dans le Phu nũ thời đàm [Chroniques des Femmes], en 1931, sur le thème «Une femme ayant perdu sa virginité peut-elle être pardonnée?», in Nguyễn Văn Ký, La Société vietnamienne face à la modernité, Paris, L'Harmattan, 1995, p. 266-267.

20. Nhất Linh, Đoạn Tuyệt [La rupture], 1935, in Văn xuôi lãng mạn Việt Nam, 1930-1945 [La littérature romanesque et romantique au Viêt Nam, 1930-1945], Hanoi, Nhà xuất bản Khoa học Xã hội, 1989, p. 55-56. 
taille soigneusement les ongles des doigts ${ }^{21}$, et on introduit son index entouré de coton dans le canal vaginal. Lorsqu'on retire le doigt, s'il est tacheté de sang, la preuve de la virginité est faite ${ }^{22}$. Cette «preuve» hémorragique reste toujours d'actualité, alors même que la liberté sexuelle est plus grande. Une jeune fille n'a plus besoin d'attendre la nuit de noces pour se risquer à perdre sa virginité. Qu'elle garde le même partenaire jusqu'à son mariage, voire au cours de sa vie, rien ne lui sera reproché. En revanche, la perte de l'hymen màng trinh restera un souvenir «inconsolable», quand elle est liée à une rupture. Une connotation péjorative s'attache à ces «blessures adolescentes » (vêt thương dậy thì), avec l'idée qu'elles sont irrémédiables, impossibles à guérir. Ce sentiment restera vivace chez l'adolescente qui a connu de telles expériences; surtout, cela va l'inhiber dans tout élan vers d'autres amours, ou bien lui dictera un comportement différent de celui des autres jeunes filles qui n'ont pas le même vécu. Une perte de la virginité qui n'aboutit pas à un mariage ou qui aboutit à un mariage avec un homme qu'on n'a pas choisi en premier, marquera le début de déboires sentimentaux, de relations coupables, d'aventures malheureuses, surtout si un enfant est né lors de ces premières relations sexuelles. L'opprobre va rejaillir non seulement sur la personne en cause, mais encore sur son enfant, de par ses origines non officiellement établies et connues ${ }^{23}$. Une «fille-mère» au Viêt Nam se voit ainsi affublée d'une appellation ignominieuse : elle est «engrossée à la sauvage» (chưa hoang) ${ }^{24}$. Cela reste toujours d'actualité. Lors de nos séjours sur le terrain dans le milieu rural au nord du Viêt Nam, dès l'entrée d'un village, on nous avertit que dans telle ou telle famille, il y a des cas de

21. Les lettrés-mandarins d'autrefois portaient des ongles non-taillés pour signifier leur mépris pour les travaux manuels.

22. D $\mathrm{D}^{\mathrm{r}}$ Nguyễn Xuân Nguyên, «Procédés sino-annamites d'expertises médico-légales », Bulletins et Travaux de l'Institut Indochinois pour l'Étude de l'homme, Hanoi, 1943, fasc. 1, p. 126. Cette citation est sidérante: violée et morte, une jeune fille saigne encore.

23. Il semble que les mœurs vietnamiennes aient évolué vers beaucoup plus de rigueur à l'égard des filles-mères. Alors que l'histoire vietnamienne a connu, au cours des onze premiers siècles, maints héros légendaires, ou fondateurs de dynasties (celles des Đinh et des Lý postérieurs, notamment), qui ont été des bâtards, cela n'a plus été le cas à partir du $\mathrm{XV}^{\mathrm{e}}$ siècle en raison de l'influence croissance du confucianisme. Cette opinion, dominante chez les historiens, mériterait toutefois un réexamen.

24. C'est, dans toute sa rudesse, la traduction littérale. Pour exprimer l'état de grossesse «normale», on utilise habituellement l'expression plus policée de có mang («avoir quelque chose à porter», «être enceinte», «être porteuse»), ou l'expression neutre de chưa, có chưa («être enceinte»). Un enfant né «sans père connu» se dit con hoang («enfant sauvage»). 
Vraies et fausses vierges au Viêt Nam. La falsification corporelle en question

chửa hoang ${ }^{25}$. Dès qu'un enfant d'une douzaine d'années vient nous accueillir, on nous prévient qu'elle est née «de père inconnu » (con hoang), devant elle, nonobstant sa grande vivacité, sa finesse, sa politesse...

\section{La perte de la virginité, son vécu}

La littérature offre deux situations significatives de cette perte. La première a pour cadre le Viêt Nam prémoderne, aux us et coutumes encore largement inspirés de la Chine ancienne, l'autre, le Viêt Nam actuel. Autre différence: dans le premier cas, Kîeu, l'hérö̈ne, est contrainte au mariage pour sauver son père; la défloration y ressemble à un viol et le proxénète exécuteur de basses œuvres envisage, à un moment, de recourir à des simulacres de la virginité pour camoufler son forfait. Dans le deuxième cas, les relations sexuelles sont libres, et libre aussi est le recours à l'hyménoplastie. Ainsi, bien qu'il s'agisse toujours de «falsifications corporelles», ces deux cas de figure comporteront de nombreuses variantes.

Écrit en caractères démotiques, le premier texte est un long roman versifié, le Kim Vân Kiêu 金雲趐, œuvre du lettré Nguyễn Du 阮做 (1765-1820) ${ }^{26}$. Grâce à sa structure et à l'agencement des rimes il est facilement mémorisable ${ }^{27}$. Des gens complètement illettrés connaissaient ses trois mille vers et étaient capables de faire des citations fort à propos en toutes circonstances de la vie quotidienne. C'était notre cas, quand, pensant louer un coloris, nous avons cité Kiêu : «Nước vỏ lựu, máu mào gà...», soit, selon nous, un beau rouge analogue « au liquide de l'écorce d'une grenade, et au sang de la crête du coq». Malheureusement, la citation se révéla suspecte. Au regard furibond de notre mère, jeune veuve fidèle à la mémoire paternelle, nous avons compris avoir, quelque part, commis une gaffe. Elle nous soumit à un véritable interrogatoire: «Où as-tu appris cela? Mais c'est une tromperie!», alors qu'à nos yeux de gamin, de douze ans à peine, cela n'avait rien de répréhensible. Lors de la lecture du Kiêu, nous avons

25. Missions sur le terrain: CNRS-Université Paris Diderot-Paris 7, 1979 (4 mois), 1982 (3 mois), 1989 (3 mois), CNRS-OMS, 1993-1994 (2 mois), 1995 (1 mois et demi), 1996-1997 (1 mois), 2000 (15 jours), UNESCO, 2003 (1 mois).

26. Le titre Kim Vân Kiêu renvoie aux noms des trois principaux personnages: Kim Trọng, le promis de Kiêu, Thúy Vân, jeune sœur de Kiêu, et Thúy Kiêuu, l'héroïne. Habituellement, on désigne l'ouvrage sous le nom abrégé de Kîeu. Sur cette œuvre, voir: http://www.nomna.org/home.php?IDcat=13 \& cat $=2$

27. Toutes les rimes sont du ton băng («égal», équivalent d'une «longue» dans la versification latine, par contraste avec les rimes trắc, équivalentes des «brèves»). Le dernier mot du vers de 6 pieds rime avec le $6^{\mathrm{e}}$ mot du vers de 8 pieds, le dernier mot du vers de 8 pieds rimera avec le dernier du vers suivant, de 6 pieds, et ainsi de suite... 
compris que ce vers décrivait une belle couleur. Combien de fois ce livre, en version bilingue, ne fut-il pas lu et relu? Dans sa traduction française, Nguyễn Văn Vĩnh, mettait en note: «Cette formule curieuse est indiquée dans le livre Bắc-lý-chí (Bei li zhi 北里誌) ${ }^{28}$. Il est relaté que les marchands d'illusions se servaient d'une infusion d'écorces de grenades et du sang de crête de coq, pour donner à leurs clients certaines illusions ${ }^{29}$.» L'explication restait elliptique alors que Nguyễn Văn Vĩnh, traducteur de talent, avait une réputation d'érudit. Bien plus tard, nous avons découvert que cette phrase traduisait son embarras, et qu'il parlait d'un procédé pour simuler la virginité ${ }^{30}$. Mais la malchance ne nous a pas quitté: le Bei li zhi, consulté dans le cadre de cette étude, ne contenait aucune allusion à ce simulacre, et il n'était question, nulle part, d'infusion d'écorces de grenade! La traduction et les commentaires de ces Anecdotes du quartier $d u$ Nord étaient l'œuvre de Robert des Rotours ${ }^{31}$ : on y trouvait, certes, un coq, mais celui-ci n'avait qu'une patte cassée, et rien n'y pouvait faire croire que l'héroïne Tchang Tchou-tchou s'en servait pour des usages intimes. Néanmoins, ce souvenir d'enfance nous a permis de faire quelques suppositions que nous soumettons humblement au lecteur, sous une forme interrogative : premièrement, comment notre mère, qui ne fréquentait pas les milieux où l'on pouvait simuler la virginité, qui ne lisait pas le Bei li zhi dans sa version érudite (où d'ailleurs il n'était question d'aucun simulacre), pouvait-elle savoir qu'il y avait quelque «tromperie» dans la phrase citée ? Fallait-il croire que cette pratique était plus connue, sinon plus répandue, et qu'on pouvait en avoir un soupçon, à demi-mot? Deuxièmement, les commentateurs vietnamiens, unanimes à faire un renvoi au Bei li zhi, en connaissaient-ils une autre version, postérieure, où était mentionné ce simulacre de la virginité, ou bien cherchaient-ils à légitimer un procédé, bien en cours dans la société vietnamienne, en prétendant le trouver dans un ouvrage chinois qui traitait de la vie des courtisanes? Ou plutôt, ne se contentaient-ils

28. Euvre de Souen K'i (Sun Qi) 孙棨 sous les T'ang. Cf. infra, note 31.

29. Nguyễn Du, Kim Vân Kîêu, traduction en français par Nguyễn Văn Vĩnh, Hanoi, Éditions Alexandre de Rhodes, 1942, p. 309, note 350.

30. Dans les Thanh lâu 青樓 [«Pavillons verts », désignation chinoise des maisons closes], on utilisait le jus de l'écorce de la grenade, substance très tannique, pour faire contracter les tissus du vagin, tandis que le sang de crête du coq, subrepticement répandu, était censé remplacer le saignement lors de la défloration. D'après Lê Văn Lân, «Chuyện y học trong Kîêu [Les questions médicales dans le Kîêu]», http://www.angelfire.com/ ks3/hodacduy0/sanchoi/sanchoi_33.htm

31. Souen K'i (Sun Qi), Courtisanes chinoises à la fin des T'ang, entre circa 789 et le 8 janvier 881. Pei-Li Tche [Anecdotes du quartier du Nord]. Traduit du chinois et annoté par Robert des Rotours, Paris, 1968 ( $c f$. chapitre XIII, Tchang Tchou-tchou, p. 156-166). Nous utiliserons l'appellation en pinyin pour désigner cet ouvrage: Bei li zhi. 
pas de copier les uns sur les autres en renvoyant l'allusion au Bei li zhi, sans jamais l'avoir lu? La question reste en suspens.

Revenons au Kî̀u. Le roman se situe quelque part en Chine, sous la dynastie des Ming. Kî̀u, une belle et talentueuse jeune fille, s'est éprise d'un jeune lettré. En l'absence de son bien-aimé, à qui elle a voué un indéfectible amour, la famille de Kiêu est victime d'une machination. Afin de sauver son père, Kiêu s'est résolue à se vendre. Pour son malheur, elle est tombée entre les mains d'un maquereau dont la mission est d'aller acheter des jeunes filles pour des lupanars. Or, l'homme, dès l'acquisition de Kiêu, ne songe qu'à la déflorer. Voici ses calculs :

Ce bon morceau tout proche

C'est notre fonds de commerce, mais comment résister quand il vous tombe du ciel?

Un tel fruit divin à portée de mes mains frustes

N'hésitons plus à le cueillir pour assouvir notre appétit.

Ici-bas, parmi les clients en quête de plaisirs

Peu savent jouir en connaisseurs.

Une mixture d'écorce de grenade et du sang de crête de coq fera l'affaire

Comme simulacre, et tout peut sembler intact.

Ce stratagème pourra tromper bien des gens peu avisés,

Nous maintiendrons nos prix, rien ne sera perdu.

Et que ma vieille patronne s'avise à me chercher noise

J'en serai quitte pour une séance de pénitence à genou ${ }^{32}$.

Pour décrire ce que ressent Kî̀u au cours de cette nuit, le poète alterne descriptions et lamentations :

Quel dommage! Cette fleur de camélia naguère si fraîche

Un bourdon, par son va et vient, la laissa dévastée.

Ce fut comme une violente bourrasque

Qui, sans pitié, ravagea ce jade précieux et ce parfum délicat.

Cette nuit du printemps, en guise de doux rêve

Sous la torche des noces, gisait la malheureuse

Pleurant à grosses larmes sa solitude,

Sa haine de l'infâme, et son corps sali :

«Quelle immonde créature

Il a souillé mon corps et déshonoré mes joues roses,

Que reste-t-il encore à espérer?

32. Kî̂u, vers 831-842. Nous utilisons l'édition de 1979, établie et commentée par Đào Duy Anh, Hanoi, Nhà xuất bản Văn học. Cette version, due à l'érudition d'un grand lettré, très curieusement, fait la part belle aux variantes de la tradition populaire (et orale). Trad. auct. 
De mon existence humaine, c'en est fini !»

Furieuse contre son destin, pleine de dépit pour son sort,

Elle saisit son couteau et pensa se suicider ${ }^{33}$.

Kiêu n'est pas pour autant quitte. Dressée au fouet par sa patronne, obligée de recevoir la clientèle, elle songe de nouveau au suicide. Un voyou l'incite à s'enfuir avec lui, mais très vite elle est reprise: il n'y aura plus d'autre issue pour elle que de se muer en véritable prostituée. Un jeune homme riche veut la racheter, mais sa femme la fait enlever par ses hommes de main pour la torturer à sa guise. Sauvée par un vaillant guerrier, elle peut enfin récompenser quelques rares bienfaiteurs, et punir les nombreux méchants. Mais ce preux est tué, victime d'un stratagème que lui tend un mandarin de la cour. L'assassin veut abuser d'elle. Devant sa résistance, il la donne en mariage à un petit fonctionnaire, originaire d'une minorité ethnique. Pour lui échapper, elle se jette dans une rivière, mais est sauvée de la noyade... Kiêu finit par retrouver sa famille et son bien-aimé.

Le deuxième cas est tiré d'un roman autobiographique paru en 2006, Thảo, du nom du personnage principal et dont l'auteure est une jeune Vietnamienne, Vũ Hoàng Hoa ${ }^{34}$. À la mort brutale de son père, disparu dans un accident, Thảo perd à la fois celui qui la protège, qui la comprend, et le tuteur de sa petite famille, composée d'une mère, actrice de théâtre, d'une grande sœur, légèrement handicapée, et d'elle-même. Elle vit, dès lors, une adolescence difficile, turbulente, anorexique. Pendant son séjour d'étude en URSS, elle tombe amoureuse d'un étudiant russe, mais se refuse à lui. Revenue à Hanoi à un moment où l'économie de marché commence à faire miroiter aux yeux des jeunes l'attrait d'une vie facile, Thảo, restée pauvre, ne tarde pas à succomber aux tentations. Elle va perdre sa virginité, presque par accident, comme une suite naturelle des flirts, «sous une table», endroit peu «romantique» et peu digne d'une «première». Très vite, elle va d'amant en amant, connaît la déception, le désabusement. Une fille qui a perdu sa virginité et connu de nombreuses aventures, à ses propres yeux, ressemblerait à des vêtements non pas hors d'usage, mais usagés, de «seconde main» ${ }^{35}$, que vont essayer d'autres jeunes, moins nantis... Jusqu'au jour où elle découvre son prince charmant, un jeune étranger. Lors de leurs rencontres, pour ne pas avoir des relations sexuelles tout de suite, elle lui avait menti: «Je suis encore vierge», dit-elle. Pour assumer jusqu'au bout ce mensonge, elle va avoir recours à l'hyménoplastie, avant de s'expatrier, et de se marier. De cette opération qui marque pour elle le début

33. Ibid., vers 845-858.

34. Vũ Hoàng Hoa, Thảo, Hanoi, Nhà xuất bản Phụ nữ, 2édition, 2007.

35. Second hand, en anglais dans le texte. Vũ Hoàng Hoa, Thảo, op. cit., p. 89 sq. 
d'une autre vie, il ne sera pas question dans le roman, juste une courte phrase pour la signaler ${ }^{36}$. Néanmoins, le mensonge lui pesait, jusqu'au jour où elle va le révéler à son mari. Thảo connaîtra sinon le bonheur parfait, du moins l'amour, tout en se délivrant d'un poids sur la conscience.

L'ouvrage, écrit dans une prose limpide, souvent poétique, décrit très bien l'attirance des jeunes pour les biens de consommation, notamment les «Hondas», ces vélomoteurs super puissants, baptisés Dream [Rêve], commercialisés par la firme Honda, et qui connaissent un très grand succès au Viêt Nam. Que ne sacrifie-t-on pas pour connaître une minute de vitesse, derrière les conducteurs de Honda? Pour échapper au regard ${ }^{37}$ inquisiteur de l'autre, qui vous fige dans votre misère matérielle, une seule solution: la richesse, l'argent! Ainsi, l'auteure a-t-elle surmonté sa pudeur, sa honte et la réprobation de sa famille pour écrire cet ouvrage autobiographique, et proposer tout au long du roman une réflexion sur la condition de jeune fille, sur la virginité. L'ouvrage connaît du succès auprès d'un jeune public, avec un certain détournement toutefois : des lycéens, des adolescents, s'envoient ce livre en guise de cadeau, à l'occasion d'un anniversaire, ou pour marquer un événement qui vous change, mais aussi pour inciter des partenaires à goûter au fruit défendu !

\section{Simulacre et hyménoplastie : les procédés}

Deux procédés de falsification de la virginité ont actuellement cours. Les appellations vietnamiennes pour les désigner sont explicites. Giả trinh, c'est «contrefaire l'hymen», ou «contrefaire la virginité», c'est le «simulacre de l'hymen». Vá trinh, désigne l'opération qui «rapièce l'hymen», ou «recoudre l'hymen», cette expression utilise le terme vá (rapiécer, recoudre), terme employé habituellement pour le «rapiéçage d'un habit (vá quầnáo)», ou pour «réparer une chambre à air» de vélo (váxăm).

Le cas de Kî̀u relève du simulacre, mais un simulacre virtuel, seulement envisagé par le proxénète, qui en énonce la composition: mixture d'écorce de grenade et sang de crête du coq. La recette d'utilisation est inconnue, certaines explications ont été tentées, plus ou moins plausibles ${ }^{38}$. L'absence de mention explicite de cette recette dans le Bei li zhi auquel renvoient tous les commentateurs vietnamiens nous interdit de faire remonter cette pratique jusqu' aux $\mathrm{IX}^{\mathrm{e}}-\mathrm{X}^{\mathrm{e}}$ siècles, en Chine, bien que ceci soit tout à fait envisageable. $\mathrm{Au}$ Viêt Nam, on peut affirmer qu'une telle pratique n'était pas inconnue dès

36. Ibid., p. 126.

37. Cái nhìn («le regard»), en italiques dans le texte, passim.

38. Voir note 30. 
le $\mathrm{XIX}^{\mathrm{e}}$ siècle. Depuis, de nombreuses variantes et modalités d'emploi sont apparues, dont la presse locale, qui donne maintes informations, toujours très prisées par le public, est très friande. La prostituée prend un bain de siège à l'eau alunée, trente minutes avant un rendez-vous, ce qui a pour effet de faire contracter le conduit vaginal, puis on y injecte un peu de sang de pigeon ${ }^{39}$ mêlé à de l'alcool ${ }^{40}$. Du sang de porc est aussi utilisé, acheté au marché, et mis sur un petit morceau de coton que la prostituée introduit au fond du vagin ${ }^{41}$. Ce sont là des méthodes artisanales et d'usage assez limité. En cas d'affluence et quand les opérations d'hyménoplastie n'ont pu être effectuées à temps, on a recours à des produits plus sophistiqués, de fabrication industrielle. Cette invention, d'origine nippone, a vite été adoptée par les Chinois et par les Vietnamiens ${ }^{42}$. L'ensemble, sous diverses appellations plus ou moins évocatrices - Artificial Virginity Hymen Kit, Virgin again, «Joan of Arc» (hommage à la «Pucelle d'Orléans ») -, est présenté dans un boîtier ou sous emballage en plastique. Outre une notice d'utilisation, il comprend une sorte de capote inversée en albumine, laquelle, introduite dans le vagin une vingtaine de minutes avant la copulation, se dilate et adhère ${ }^{43}$ au conduit vaginal. Lors de l'acte sexuel, elle délivrera un liquide rouge, ersatz du sang de la défloration. Comment expliquer le succès de ce kit? Bon marché - il coûte environ trente dollars - et fonctionnel, il ne nécessite aucune intervention spéciale; tel que, il est censé falsifier la virginité, non par le remplacement de l'hymen déchiré, mais par la production du signe extérieur le plus fréquemment admis en cas de défloration: l'hémorragie. Au Viêt Nam, la presse féminine se déchaîne contre son emploi en avançant des arguments médicaux, traduits par ailleurs de la presse chinoise: nul ne connaît la composition de ce liquide rougeâtre, parfois fétide; il peut se produire des réactions d'intolérance pour celle qui l'utilise, comme pour son partenaire. D'autres séquelles, plus graves, sont évoquées: la stérilité pour l'utilisatrice, mais la cause de ce risque n'est pas établie. On notera que l'ensemble des simulacres, ainsi que le «kit» d'invention relativement récente, s'adressent, en général, à des prostituées. Ces dernières ont l'obligation de simuler la virginité pour attirer la clientèle, autant de fois et aussi rapidement que possible. Un tel procédé n'a pas vocation à être utilisé dans des cas d'union durable, en vue d'un

39. Le sang de poisson, qui «ne coagule pas», est aussi employé.

40. En 1992, une maquerelle à Hochiminhville a été condamnée pour de telles pratiques.

41. Autre cas relaté en 2008, source «Vie familiale sur Internet», http://giadinh.net. vn/20081030081315796 p0c1000/cong-nghe-lam-trinh-gia. htm

42. Pour information, image, prix, sur Internet, tapez: Gigimo. (Cette note est libre de toute publicité).

43. L'adhérence n'est que théorique, on verra un peu plus loin ce qui peut arriver. 
mariage par exemple, ou lorsque les prostituées ont affaire à une clientèle plus exigeante. Il sera remplacé par l'hyménoplastie.

L'hyménoplastie contrefait la virginité d'une manière plus radicale, en recousant l'hymen. Néanmoins, celui qui la pratique, le chirurgien, ne se sent pas forcément l'âme d'un falsificateur. Il «répare», tel un artisan, ce qu'on lui demande de réparer. Seules les techniques chirurgicales permettent une telle réparation. Sous anesthésie locale, l'intervention est effectuée, la plupart du temps, avec les garanties d'asepsie par un personnel qualifié. Cette «médicalisation» présente un avantage: elle rend acceptable la présence d'une personne étrangère, parfois du sexe masculin, pour une intervention dans l'espace intime de la femme. L'une d'elles a accepté de répondre à nos questions ${ }^{44}$.

Q. : Comment avez-vous pu trouver un (ou une) chirurgien(ne) pour pratiquer l'hyménoplastie?

R. : Une de mes cousines lointaines pratique la chirurgie esthétique à Saigon. Des membres de ma famille sont venus lui demander de rectifier qui son nez, qui ses paupières... J'ai pris rendez-vous avec elle, la date optimale est quelques jours après les règles.

Q.: Comment s'est passée l'entrevue? Vous étiez accompagnée?

R.: Je suis venue à cette clinique toute seule, vêtue d'une jupe à fleurs. La fille de la chirurgienne, qui m'a accueillie, m'a félicitée: «Tu as bien fait de venir en jupe, il suffit de la relever lors de l'opération». Effectivement, sur le billard, je l'ai relevée. Des lampes de différentes tailles étaient dirigées vers cet endroit. J'ai reçu des sédatifs et quelques piqûres d'anesthésie locale. Les lampes étaient braquées sur ma nudité.

Q.: C'est ce qui vous a choqué le plus?

R. : Non. La chirurgienne s'est exclamée: «Dis donc, comme c'est large! As-tu pratiqué un curetage de l'utérus (nạo thai, «pratiquer un avortement»)? - Jamais, madame. Sa fille m'a jeté un regard apitoyé. Jamais je ne me suis sentie aussi dévalorisée, ravalée au bas de l'échelle sociale, avilie. Mais ce n’est pas tout. «Même des prostituées viennent ici se faire recoudre leur hymen, ma fille, ajouta-t-elle... Ce n'est rien, c'est indolore, et ce sera fini tout de suite !». Je ravale mes larmes, je ne suis donc qu' une prostituée, cette «largeur» c'est celle de la prostitution, quelle honte!

Je n'ai presque rien senti. Je me suis représentée un travail de brodeuse qui rapièce un dessous de service à thé, une sorte de toile d'araignée. Sous l'effet de l'émotion et du sédatif, je tombe dans un court sommeil.

44. Lors de notre mission en 1995, nous avons rencontré cette jeune informatrice. Mais l'interview n'a été effectuée que plus tard, pour les besoins de cette étude (10/05/2008). Du long entretien qui en résulte nous reproduisons seulement la partie qui concerne l'hyménoplastie. Traduction de l'auteur. 


\section{Đinh Trọng Hiếu}

La chirurgienne: «Ça y est, vraiment bien, je t'ai refait une fleur virginale ! Veux-tu voir?». Comment? me dis-je, voir «ça» dans un miroir? Je ne me suis jamais vue là, je n'ose pas m'y regarder; finalement je n'ai rien vu. Mais j'ai l'impression que ma porte, désormais, sera protégée par un rideau de dentelles à fleurs, évidemment de couleur blanche, et dont la broderie m'a été faite avec du fil résorbable...

Q. : Combien ça vous a coûté?

R. : «Cela coûte 160000 đông ${ }^{45}$ », a répondu la chirurgienne à ma question. «C'est le tarif pour les proches, dit elle, je ne te prends que le coût des médicaments».

Q. : Cela vous a-t-il fait mal?

R.: Non. Mais par la suite j'ai ressenti une douleur lancinante. J'ai un peu saigné, c'est comme si j'avais de nouveau mes règles. Je me déplace avec difficultés et dois prendre des antibiotiques. Après un certain temps, j'ai pris l'avion pour revenir à Hanoi.

Q.: Qu'avez-vous ressenti?

R.: Quand la plaie s'est cicatrisée, un sentiment bizarre, de fierté, m'a saisie. Désormais, une fleur blanche a comblé ce trou béant. Cette fleur virginale a enterré les souvenirs désagréables au fond d'un abîme, dans une tombe, avec tout un passé. Je suis redevenue pure. Je suis sortie victorieuse et plane maintenant sur la tête de tous ces hommes, fantômes d'un passé dégoûtant. Je suis redevenue pure, blanche, et je réserverai cette pureté pour celui que j' aime. À travers cette membrane renouvelée, je me suis rendu justice à moi-même. Les souffrances du passé sont annihilées... Néanmoins, cette fleur recousue, même avec du fil résorbable, reste en relief, dans ma pensée. Elle m'est étrangère et n'arrive pas à se fondre en moi-même. C'est pourquoi elle me gêne, elle m'obsède... Bizarrement, parce que je suis redevenue vierge, il semble que mon futur mari me respecte davantage; lors de nos rencontres, il ne touche plus à cette partie... Nos liens se resserrent de plus en plus, le jour du mariage approche. Je suis plus sûre de moi-même, comme quelqu'un qui va passer un examen, muni du meilleur des bagages...

Q.: Y-a-t-il eu saignement?

R. : J'ai saigné lors des rapports, beaucoup, ça m'a fait mal...

Cette informatrice est l'une des rares à avoir surmonté ses appréhensions pour nous raconter un pénible vécu. Nous avons voulu savoir par quel réseau elle a pu parvenir à se faire opérer, car ce n'était pas toujours facile, surtout en 1995. Finalement, c'était un réseau familial, qui fonctionne de bouche à oreille. Actuellement, la publicité, notamment sur Internet, rend caducs ces réseaux d'antan: se faire recoudre l'hymen, à condition de pouvoir payer, est plus facile

45. 160000 đông, en 1995, valent à peu près une vingtaine de dollars, ce qui, en effet, est bon marché. 
que se faire soigner de maladies ordinaires ${ }^{46}$. La médicalisation rend acceptable non seulement une opération à connotation éthique, mais encore une présence étrangère, la chirurgienne devenant cette «brodeuse» qui transforme, presque magiquement, une souillure en pureté. Néanmoins, nettement, le même objet naturel qu'est le vagin est perçu: d'abord comme un objet (toute la réalité est renvoyée, brutalement: ce vagin est large, comme celui d'une prostituée); il réintègre finalement le corps de la personne, qui, tout en refusant de le voir, l'entoure de représentations imaginaires. Le paiement, la honte, la souffrance postopératoire, tout cela fait partie du rachat et de l'expiation, qui rendent possible cette «pureté » retrouvée. Signalons cependant qu'il s'agit d'un cas «optimal» : la personne va faire une opération d'hyménoplastie volontairement, elle n'y est pas contrainte pour se livrer au commerce de son corps; la chirurgienne, malgré son langage brutal, est expérimentée et efficace. L'opération est réussie. Tout ne se déroule pas toujours dans d'aussi bonnes conditions. Nous aborderons plus tard le cas où, après le mariage, le mari découvre ce stratagème.

Nous avons posé à un chirurgien chevronné et ami de longue date ${ }^{47}$ toute une série de questions. Voici ses réponses, traduites du vietnamien:

L'hymen ne peut être perçu que par auscultation visuelle, on ne sent presque rien par le toucher ou lors de la pénétration du pénis. Par contre, sa déchirure provoque une hémorragie: quelques gouttes de sang, un peu davantage si les rapports sont violents. L'hyménoplastie est une opération délicate, plus délicate que les autres opérations intimes. Elle ne répond pas toujours à toutes les espérances, parce qu'il n'y a pratiquement plus de vaisseaux sanguins sur les parties restantes qui vont servir de support à la réfection. En général et en pratique, nous rebouchons le vagin, pour ainsi dire, avec les restes de membranes. L'opération sera facilitée si la déchirure est de fraîche date et incomplète; dans le cas contraire, elle est difficile. Tant et si bien que lors des relations sexuelles survenues après l'opération, il n'y a pas forcément les quelques gouttes de sang escomptées, et qui constituent la preuve communément admise d'une «virginité». L'habileté du chirurgien est d'inclure,

46. Dans une revue dénommée Le Monde culturel, de soixante-trois pages, on dénombre dix pleines pages de suppléments publicitaires consacrés aux cliniques privées spécialisées en «esthétique». Thế giới văn hóa, n 18 du 14/05/2008.

47. C'est cette amitié qui nous permet d'aborder des sujets peu fréquents dans les conversations, et d'avoir quelques indications sur des «tours de main» techniques. L'anonymat est requis ici car la législation en vigueur interdit toute publicité quand il s'agit d'actes médicaux. Autre précision: cet ami chirurgien, d'origine vietnamienne, exerce dans un pays démocratique, et n'a pas à pratiquer des opérations d'hyménoplastie cauteleuses. L'hyménoplastie fait partie de la chirurgie réparatrice. Les opérations sont réitérées quand il y a une demande répétée émanant des patientes. Ce n'est pas le rôle du chirurgien d'aller s'enquérir de leurs motivations. Bien que parfois difficile à admettre, la liberté du corps féminin fait partie intégrante de la liberté. Entretien du 17/03/2009. 
lors de l'opération pour recoudre ces membranes, des parties avec capillaires, ce qui va provoquer l'hémorragie et la satisfaction pour ceux (ou celles) qui recherchent cette sensation. Autrement, l'intégralité de l'hymen n'est pas perceptible, sauf par auscultation visuelle.

Q.: Vous est-il arrivé de pratiquer l'hyménoplastie plusieurs fois de suite sur une même personne?

R. : Oui, plus l'opération est difficile, plus c'est un défi pour notre habileté. Nous essayons de répondre au mieux à l'attente de nos patientes et nous ne pratiquons une opération qu'après avoir mis celles-ci en parfaite confiance. Entre nous et elles, il y a comme une sorte de «complicité» et une opération doit se dérouler avec le maximum de confort, sans aucune douleur, appréhension, ni sentiment de honte ou culpabilité...

\section{La réaction après la défloration}

Comment réagissent les hommes, auteurs de la défloration? Van Gulik, dans son ouvrage sur La Vie sexuelle dans la Chine ancienne, relate l'une de ces réactions: «Le Tcho-keng-eou de source Yuan, donne dans son chapitre 28 un poème [...] qui se réfère à cette coutume (de garder la serviette tachée du sang de la défloration); il fut dédié à un homme qui, la nuit de ses noces, découvrit que son épousée n'était plus vierge.

Cette nuit, une magnifique noce a eu lieu;

Mais quand je me suis mis en devoir d'explorer la fleur parfumée,

J'ai découvert que le printemps était déjà passé par là.

Beaucoup de rouge, peu de rouge, à quoi bon en demander tant?

Il n'y a rien à voir, non, rien à voir !

Je vous renvoie le coupon de soie blanche ${ }^{48}$.

L'autre réaction est extraite du roman $T_{h a ̉ o}{ }^{49}$. C'est le mari qui parle, sa jeune femme vient de lui avouer qu'elle a eu recours à l'hyménoplastie pour simuler la virginité :

Ma petite, comme tu es naïve, sourit Bastien. Tu ne sais pas à quel point tu étais adorable et captivante le jour de notre rencontre. Combien avons-nous été heureux ensemble! Ta rencontre a apporté quelque chose de merveilleux à mon existence. À tes côtés, je connais le bonheur. Je suis parti vers ce bonheur, répondant à cet appel, instinctivement. En définitive, tu me parais très rétrograde. Quelle injustice et quelle sottise, un mariage qui serait subordonné à la virginité de la femme. Sais-tu que nous vivons au XXI ${ }^{\mathrm{e}}$ siècle? La virginité n'a plus d'existence, elle est dépourvue de sens.

48. Op. cit., p. 264.

49. Vũ Hoàng Hoa, op. cit. 
Vraies et fausses vierges au Viêt Nam. La falsification corporelle en question

Il faudrait être très étroit d'esprit et tout à fait passéiste pour maintenir ce préjugé qui nous oblige à lier notre bonheur à l'intégralité de cette membrane : l'hymen [...]. Cette membrane n'a aucune signification ${ }^{50}$.

Les deux dernières réactions sont dues à la plume de l'écrivain chinois Yu Hua. Son roman Brothers ${ }^{51}$ décrit avec truculence les mésaventures d'un potentat local, le directeur Li Guangtou. En tant qu'organisateur d'un concours de beauté pour trois mille miss «vierges», il estimait être en droit d'en connaître une authentique. Il ordonna à son attaché de presse de lui en amener une belle, de grande taille, miss 1358. Le lendemain, comprenant finalement que miss 1358 avait subi une réfection de l'hymen, pour paraitre vierge, il s'écria:

- Putain, je me suis fait blouser!

Liu l'Attaché de presse sursauta et ouvrit des yeux ronds.

- Elle a déjà eu un enfant, ajouta Li Guangtou. Elle a des vergetures de grossesse sur le ventre. Putain, elle s'est sûrement fait restaurer l'hymen. Putain, ce n'était pas un produit d'origine, c'était du bricolé... ${ }^{52}$

Autre mésaventure quand on lui amena, le lendemain, la numéro 864 :

Ce que Liu l'Attaché de presse ignorait, c'est que cette numéro 864 n'était plus une vierge d'origine depuis longtemps, et qu'elle n'était même pas une vierge bricolée. C'était plutôt une vierge discount. Pour remporter la palme, elle avait déjà couché avec six des jurés, et elle avait acheté successivement au charlatan Zhou You six hymens artificiels importés de la marque Sainte Jeanne d'Arc ${ }^{53}$. Par six fois, le sang avait coulé, et chacun des six jurés, abusé, avait cru posséder une vierge. Son cas était encore bien pire que celui de la numéro 1358, car si cette dernière s'était fait restaurer l'hymen, au moins avait-elle gardé sa virginité bricolée jusqu'à ce qu'elle arrive dans le lit de Li Guangtou ${ }^{54}$.

Malheureusement, comme la numéro 864 s'était adressée à Zhou You pour acquérir l'hymen artificiel, et qu'il ne reste qu'un exemplaire d'une marque locale «Meng Jiangnü», celui-ci s'est décollé pendant les ébats. Li Guangtou annonça à son attaché de presse :

- C'était encore de la marchandise frelatée, de l'artificiel. Putain, il est même ressorti.

50. Op. cit., p. 200.

51. Yu Hua, Brothers, roman traduit du chinois par Angel Pino et Isabelle Rabut, Arles, Actes Sud, 2008.

52. Ibid., p. 569.

53. Bien que de fabrication asiatique, ce produit porte le nom de «Joan of Arc», avec une pointe d'humour so British.

54. Yu Hua, Brothers, op. cit., p. 570. 
Li Guangtou expliqua qu'au moment de la pénétration, il avait senti quelque chose de bizarre. Il recourut à une métaphore:

- C'est comme quand on met le pied dans une chaussure où il y a une chaussette, on sent quelque chose qui gêne ${ }^{55}$.

Trois époques, trois discours. Le premier dénote une attitude désinvolte, contre mauvaise fortune bon cœur, c'est un langage empreint de poésie, sinon d'humour. Le deuxième, celui d'un intellectuel qui cherche à convaincre par le sentiment et par la raison, convient à quelqu'un qui aime une Vietnamienne, mais qui ignore tout de la pression sociale dont celle-ci est victime. Le troisième discours, parsemé de grossièretés, est celui, remarquablement rendu, d'un potentat. Le texte donne non seulement des informations sur les acteurs, mais aussi sur les aléas de la falsification: l'exactitude des observations de Yu Hua est frappante: un hymen vierge ne peut être constaté que de visu, c'est à cette fin qu'on avait doté Li Guangtou d'un microscope ${ }^{56}$, de jumelles et d'une loupe, pour qu'il procède à la vérification de ses miss. La miss 1368, qui s'est offert une hyménoplastie, certainement mal exécutée, n'a pas saigné à l'œuvre ; elle se désole d'ailleurs de ce choix qui s'avère moins «flexible»: «L'hymen qu'elle s'était fait refaire à prix d'or ne lui avait servi à rien jusqu'à présent, tandis que ces hymens artificiels bon marché faisaient fureur ${ }^{57}$ ». Même exactitude d'observation (et de description) à propos du dysfonctionnement de l'hymen artificiel de la marque locale «Meng Jiangnü»... Bien que Yu Hua décrive la société chinoise, ses observations sont tout à fait transposables au Viêt Nam du fait de la mondialisation et de la proximité culturelle et géographique entre les deux pays. Nombre de cars de touristes déchargent au Viêt Nam des émules de Li Guangtou, c'est un spectacle fréquent, surtout dans les villes frontalières ${ }^{58}$.

\section{Falsifications corporelles}

La contrefaçon de la virginité fait partie des falsifications corporelles. Parmi ces dernières, toutes ne sont pas traumatisantes: un sosie imite un modèle plus ou moins prestigieux, un mime ou un acteur de théâtre utilise ses mimiques corporelles, son «jeu», sa gestuelle, pour s'exprimer, dans le cadre d'une prestation artistique. On ne saurait dire la même chose de celles qui ont recours à la falsification pour paraître vierges.

55. Ibid., p. 573.

56. Le microscope fait partie de l'exagération stylistique destinée à forcer la caricature des gens obséquieux et incultes qui gravitent autour du nouveau pouvoir.

57. Yu Hua, Brothers, op. cit., p. 563.

58. À Cao Bằng, Lạng Sơn, Móng Cái, notamment. 
La falsification peut avoir un but non vénal, c'est celui des jeunes filles qui veulent contracter un mariage honorable et durable, alors qu'elles ont perdu leur virginité. Dans ce cas, le recours à l'hyménoplastie est fréquent, et la «supercherie» réussit dans la plupart des cas. Le bonheur conjugal peut exister, le chirurgien peut estimer qu'il y a contribué. Même quand la femme, après le mariage, reprend son assurance et avoue au mari la supercherie, comme l'a fait Thảo, le couple peut durer. Il s'agit cependant d'exceptions. La plupart du temps, une femme qui a recours à ce stratagème avant de contracter un mariage, vit dans une situation d'instabilité. Car, à moins de s'expatrier, elle risque quotidiennement d'être dénoncée par d'anciens amants rendus jaloux, par des voisins et voisines mal intentionnés. Alors, le mariage ne sera plus celui escompté par la femme: il y aura rupture, ou bien on tombe dans le cas de ces unions boiteuses, où l'homme est investi de tous les droits, alors que la femme, fautive, doit courber la tête et accepter n'importe quelle vexation, n'importe quelle injustice. Au Viêt Nam, où les femmes sont plus nombreuses que les hommes ${ }^{59}$, le mari se donne le droit d'avoir des relations extra-conjugales, d'avoir des concubines, alors que la femme, pour éviter la rupture, toujours mal vue socialement, doit fermer les yeux. La situation est analogue pour une fille-mère qui veut se marier, elle doit renoncer à son enfant si elle veut fonder un autre foyer ${ }^{60}$. Ainsi, malgré l'égalité proclamée entre homme et femme, malgré les «lois protégeant la femme et l'enfant», malgré l'absence de toute «loi religieuse », bouddhique et/ou catholique, interdisant qu'un homme puisse prendre comme femme une non-vierge, la règle générale reste qu'une vierge est préférée à son contraire. On assiste à un «intégrisme» rampant qui prône virginité et fidélité pour la femme, et qui fait qu'une femme non-vierge reste paria au milieu de ses semblables. Pour échapper à cette situation, les jeunes de nos jours, aux mœurs plus «libérées», se choisissent dès leur jeune âge, le plus souvent avec la bénédiction des parents. Un garçon pratique ce qu'on appelle le giâm ${ }^{61}$, «mûrissement sous protection», qui n'est pas sans rappeler le comportement d'Arnolphe dans L'École des Femmes du temps de Molière. Ils peuvent avoir des relations sexuelles avant le mariage, et quand, enfin, le garçon se marie avec sa partenaire, il est assuré d'avoir épousé une jeune femme «vierge ».

59. D'après le recensement de 1999, on note une masculinité actuellement plus grande au Viêt Nam, à la naissance (Voir Isabelle Attané, En espérant un fils, Paris, INED, 2010, p. 98); néanmoins cela ne change en rien les données précédentes, puisque les garçonnets en excédent n'ont que onze ans.

60. Parmi les enfants adoptés, d'origine vietnamienne, un certain nombre ont été abandonnés par une mère, obligée de le faire pour pouvoir «refaire sa vie».

61. Terme technique qui signifie «faire mûrir un fruit quand on le cueille vert». 
Ces pratiques constituent la survivance des règles d'encadrement d'une société patriarcale, où la pratique du culte des ancêtres reste ancrée et est, de loin, la plus répandue ${ }^{62}$. Dans l'ancien temps, quand une femme était convaincue d'adultère, la part de l'«encens et du feu» (hưo’ng hỏa香火) risquait d'être remise en question et de passer à une branche cadette. Or, cette part constituait la part léonine dans l'héritage familial de jadis. C'est d'ailleurs encore le cas de nos jours. Si quelqu'un se marie avec une femme non-vierge, qui peut garantir qu'elle ne porte pas un embryon d'enfant lors de ses premières relations sexuelles? Cet embryon, dont la présence est difficilement décelable, sauf par l'absence des règles, va naître dans le nouveau foyer et sera considéré comme enfant officiel par le mari, en toute bonne foi. Si personne n'est au courant de cette (fausse) filiation (hormis la mère), et si l'enfant est un garçon, il sera l'aîné et bénéficiera de la part de l' «encens et du feu», il honorera son (faux) père et sa (vraie) mère, comme selon les coutumes. Mais si la supercherie (et il y a eu supercherie, puisque le mari est dans l'ignorance) est découverte, la femme est considérée comme adultère. Mais il y a pire: quand l'enfant veut rechercher les restes de son père, et le demande à sa mère, notamment pour pouvoir les enterrer dans un site faste d'un point de vue géomantique, sa mère va lui indiquer qui est son vrai père, et où se trouvent ses restes ${ }^{63}$; dès lors, l'enfant découvre qui est son véritable géniteur, et c'est la mémoire de celui-ci qu'il va honorer, et non la mémoire de son père nourricier ${ }^{64}$. La meilleure garantie, pour un mari, que sa descendance soit biologiquement pure est d'épouser une jeune fille vierge, et qu'elle lui soit, sa vie durant, fidèle. C'est le sens du titre de trinh tiết ${ }^{65}$ dont on affublait (et affuble encore) une femme «vertueuse» au Viêt Nam...

\section{Commerce du sexe et «virginité»}

Actuellement, la «fabrication» d'une fausse vierge requiert un peu plus de doigté : ceux (ou celles) qui dirigent les lupanars modernes opèrent un choix entre les prostituées, pour sélectionner des silhouettes graciles, censées être plus proches des «vierges». Celles-ci effectuent également un rapide stage de

62. Voir, à ce propos, notre article: Đinh Trọng Hiêu, « Rythmes des vivants, mémoire des morts. Espace, temps, rituels du culte des ancêtres», in «Populations du Sud-Est asiatique», Hommes \& Migrations, juillet 1990, p. 17-26.

63. Car il ne sert à rien d'enterrer les restes d'un père nourricier sur un site intéressant du point de vue géomantique. Les effets fastes ne rejailliront jamais sur l'enfant adopté.

64. C'est ce qui est décrit dans la «Légende de Đinh Bộ Lĩnh ». Selon cette légende, Đinh Bộ Lĩnh, enfant bâtard, fut adopté par Trân Lãm. Plus tard, Đinh Bộ Lĩnh va fonder la dynastie des Đinh ( $\mathrm{x}^{\mathrm{e}}$ siècle).

65. Voir supra. 
gestes, cris et mimiques, pour simuler l'inexpérience, l'innocence, la pudeur. Généralement, sous la pression des proxénètes, dans les lupanars «bas de gamme», ces prostituées utilisent des hymens artificiels, à destination d'une clientèle peu regardante. Pour des clients plus exigeants, on offre des fausses vierges qui ont recours à l'hyménoplastie, pratique qui exige plus de temps, de technicité, et coûte beaucoup plus cher ${ }^{66}$. Rares sont ceux qui peuvent goûter aux joies de pouvoir déflorer une vraie vierge. Celle-ci existe, au Viêt Nam, comme partout dans le monde. L'attitude de Li Guangtou qui se désole de ne plus trouver de vierges relève de l'exagération littéraire: "Il n'y avait pas une seule vierge aujourd'hui dans les rues, il n'y en avait plus que dans les jardins d'enfants ${ }^{67}$.» Seulement, il faut du temps pour dénicher cette denrée rare, il faut savoir parler et plaire à ces jeunes filles, souvent capricieuses. Or, ceux qui cherchent à déflorer une vierge n'ont pas le temps, ne savent plus conter fleurette, en revanche, ils ont de l'argent, et parfois plus que de l'argent, ils ont le pouvoir. Ceux-ci peuvent rencontrer une vraie vierge. Généralement, celle-ci est trompée par ses amies, par son entourage, ou pressée par des besoins d'argent afin d'éponger une dette. Elle est destinée à une clientèle locale: commerçants enrichis qui peuvent couvrir les intermédiaires au cas où il y a plainte de la part de la victime, ou de sa famille ${ }^{68}$. Des réseaux permettent aux utilisateurs de remonter jusqu'à leur proie, et beaucoup d'intermédiaires n'hésitent pas à solliciter directement des familles dans le besoin, pour qu'elles vendent leur(s) jeune(s) fille(s) vierge(s). Le prix à payer est énorme, mais il y a une double sécurité : généralement ces vierges sont «d'origine $(z i n \text {, en argot vietnamien })^{69}$, ensuite on ne risque pas d'avoir des poursuites judiciaires, ces vraies vierges étant «consentantes».

Quand on excepte les cas de falsification de virginité en vue d'un mariage, d'une union durable, les autres utilisatrices de ces subterfuges sont des prostituées. Il y a là un curieux paradoxe: des clients viennent dans les lupanars, soit des lieux où l'on pratique le commerce du sexe, à la recherche des vierges! Cette

66. Au tarif actuellement pratiqué, une hyménoplastie coûte plus de deux millions de đông. Pour déflorer une telle «vierge», le client doit débourser, au minimum, six millions de đông. Une fois défalqué le prix de l'hyménoplastie, la prostituée recevra un million, le solde va aux organisateurs. La tentation est grande de faire faire des opérations à la chaîne et à bas prix par des charlatans, pour en diminuer le coût.

67. Yu Hua, Brothers, op. cit., p. 575.

68. Le Thế giới Phu nũ [Le Monde des Femmes] du 13 avril 2009, indique le prix d'un dépucelage en 2009: dix-sept millions de đông. Tous les intermédiaires ont été traduits devant la justice, pour «incitation à la prostitution». Mais il n'y a aucune mention du verdict.

69. Comme un produit «garanti d'origine». 
quête relève, dès lors, du commerce sexuel, tout en le transcendant quelque peu. Nous connaissons bien, maintenant, l'attrait des touristes sexuels pour les jeunes Asiatiques, il y a une publication assez volumineuse sur le sujet, au titre évocateur: Voyage au bout du sexe ${ }^{70}$ ! Néanmoins, il n'y est nullement question de recherche de vierges à déflorer, sauf pour éviter d'attraper le sida, car l'ouvrage est rédigé dans une optique globale des relations «Nord-Sud». Sans dénier le caractère «inégalitaire» de ces relations, et, en particulier, du tourisme sexuel en Asie (= Sud) pratiqué par des Occidentaux (= Nord), il y a un aspect culturel qui n'y est pas pris en compte. Un Occidental partira à la recherche d'une partenaire agréable, mais pas forcément vierge. Le commerce du sexe se limite au sexe, et pas au-delà. Comment donc expliquer cette attirance toute asiatique des hommes pour la virginité, jusqu'au point où cela devient source de commerce?

Une explication «démographique» à ce phénomène peut être tentée. Comme l'Inde, la Chine connaît un excédent d'hommes. Quand il y a polyandrie forcée, quand toute une fratrie se partage la même femme, par exemple, il y a forcément une frustration de la part de ceux qui n'ont pas connu la défloration, surtout quand l'imagination virile la pare d'attraits «paradisiaques ». Il y a certainement aussi une dimension socio-économique : les hommes, dans les pays à économie montante, grands ou petits Tigres, grands ou petits Dragons, travaillent dur, ils n'ont pas le temps de découvrir le sentiment de l'amour, ou tout simplement, l'autre sexe. En revanche, ils ont de l'argent, et/ou le pouvoir. Des séjours de détente sont organisés comme «loisirs », mais leur temps est limité, le recours à de vrais «extras » s'opère dans le cadre des réseaux du commerce sexuel: ils vont dans les lupanars et exigent une vierge! L'écrivain Yu Hua, faisant le portrait du potentat Li Guangtou, nous en donne un modèle du genre, mais pour lui, vu son importance, on conduit la proie à domicile:

Le soir, à 20 heures tapantes, Liu l'attaché de presse conduisit en personne la numéro 1358 jusqu'à la somptueuse résidence de Li Guangtou. Il l'introduisit dans la chambre à coucher de ce dernier, puis se retira. Li Guangtou avait déjà pris son bain, et il était nu sous son pyjama. Assis dans un fauteuil, il regardait la télévision. Quand il vit entrer la numéro 1358, songeant qu'elle était vierge, il se promit de se conduire en gentleman. Il éteignit la télévision et se leva, puis il s'inclina devant la numéro 1358. Il aurait voulu prononcer devant elle quelques propos galants, mais les mots lui manquèrent. Il frappa rageusement son crâne rasé :

- Putain, je suis incapable de parler d'amour ${ }^{71}$.

70. Franck Michel, Voyage au bout du sexe. Trafics et tourismes sexuels en Asie et ailleurs, Sainte-Foix PQ, Presses de l'Université de Laval, «Nord-Sud», 2006.

71. Yu Hua, Brothers, op. cit., p. 565. 
D'autres motivations existent, qui émanent toujours des gens nantis, mais qui relèvent du domaine des croyances, dont l'origine est à chercher dans ces pouvoirs «surnaturels» prêtés aux vierges ${ }^{72}$. Les déflorer donne de la chance, ou peut sortir quelqu'un d'une mauvaise passe. C'est ainsi que des négociants asiatiques, avant de conclure un très gros contrat, ou des joueurs, avant une mise importante, s'offrent la défloration d'une vierge. Pour améliorer leur confort et stimuler leur ardeur, toute une gamme d'élixirs et/ou d'aphrodisiaques est proposée : élixir de gecko, élixir de serpents, alcool où palpite un cœur de reptile, bile d'ours soutirée in vivo de l'animal ${ }^{73}$, etc. ; il s'agit là d'un ensemble d'artifices collatéraux qui servent de logistique à l'industrie du tourisme sexuel. Citons, en dernier lieu, un type de raisonnement simpliste : une vierge étant, par définition, quelqu'un qui n'a pas eu de relations sexuelles, elle est donc exempte de «maladies sexuellement transmissibles », notamment du sida; comme elle est dotée, en plus, de «pouvoirs», la déflorer peut guérir de cette maladie redoutable. Ce raisonnement conduit les riches malades atteints du sida, à chercher à déflorer une vierge ${ }^{74}$. L'entreprise est d'autant plus criminelle que la vierge, vraie ou fausse, qui ne pense pas à se protéger, ou qui n'en a pas le droit, peut être gravement contaminée à son insu.

Au Viêt Nam, aucune loi ne punit la contrefaçon de la virginité, et on ne connaît pas, non plus, des cas où les clients trompés par cette falsification, quand ils la découvrent, intenteraient un procès pour «usage de faux». Est-ce une carence du droit, en particulier, ou est-ce plutôt dû à ce que nous ne sommes pas en présence de ce qu'on désigne par «État de droit»? En effet, les objets vrais ou faux ont, de tous temps, existé, circulé, sans que personne ne s'émeuve. Le cadre d'une culture qui laisse à la perspicacité de chacun le soin de distinguer le vrai du faux aggrave la situation. Si vous avez acquis un faux objet, une fausse denrée, la faute vous incombe, il faudrait être plus vigilant, plus perspicace, la fois suivante; c'est en ayant tâté du faux qu'on apprend à reconnaître le vrai. Dans le passé, seuls les fabricants de fausses monnaies étaient châtiés.

72. Dans les sources de la tradition orale, il est toujours question d'immoler une vierge aux génies malfaisants, ou d'emmurer vivantes des vierges pour qu'elles deviennent de redoutables gardiennes de trésors. Dans d'autres traditions, on immole ou honore des vierges, certainement à cause de leur «pureté».

73. Voir Courrier international, cité dans Direct Matin (01/04/2010), p. 20.

74. C'est le même raisonnement qui conduit ceux qui recherchent les plaisirs sexuels, tout en redoutant la contamination du sida, à jeter leur dévolu sur des... enfants. Nous ne traitons pas, ici, de cet aspect particulier qui relève aussi de la pédophilie. Cf. Voyage au bout du sexe, op. cit., p. 289. Voir aussi: Alain Epelboin, «Le "sacre" de la virginité, d'hier à aujourd'hui», interview (07/09/2007) par Habibou Bangré: http://www.afrik. com/article 12417.html 
Sous le régime colonial, pour lutter contre les falsifications, le dépôt d'une marque a été institué. Sur certains produits de cette période on pouvait lire une curieuse inscription: «Nhãn hiệu trình toà », soit l'équivalent de la mention, quasi internationale, de «Trade mark» ou «Marque déposée », mais rebaptisée pompeusement en vietnamien «Marque déposée au tribunal». De nos jours, le Viêt Nam, qui est environné par des nations bien rodées dans l'industrie du faux, porte l'essentiel de ses efforts dans la lutte contre la circulation et l'utilisation des faux médicaments. On n'a même pas besoin de sévir contre les faux-monnayeurs, sauf contre la circulation de faux dollars, lesquels rapportent bien plus qu'une éventuelle fausse monnaie locale. Dans ce contexte, la virginité, qui n'a jamais bénéficié d'un «label déposé au tribunal», peut être librement falsifiée. On punit, non pas cette contrefaçon, mais des maquerelles et proxénètes, maillons parfois visibles de réseaux souterrains, aux ramifications nationales ou multinationales. L'enquêteur peut se rendre compte assez aisément du phénomène en écoutant simplement le bavardage des prostituées : à leur accent, on sait qu'elles ont été déplacées en masse des villages du delta du Mékong pour aller exercer au Nord, des villages du Nord pour aller exercer au Cambodge... Depuis longtemps toute pudibonderie idéologique, toute référence morale à l' «homme nouveau» a été bannie. À l'ère de l'économie de marché, vraies ou fausses, les vierges rapportent... C'est l'essentiel.

Se dessinent, dès lors, des contours assez flous, mais qui forment la périphérie des pays - Dragons (et Tigres) plus ou moins grands - d'un espace où fleurit le commerce du sexe. Bien que les Vietnamiennes forment le plus gros de la troupe $^{75}$, on assiste à une «internationalisation» du phénomène. Jusqu'à plus

75. Ne peut-on pas faire un parallèle avec le cas des «mariages arrangés», sur lesquels nous avons quelques données? Selon le journal vietnamien Tiên Phong («Avant-garde»), l'argument de virginité constitue un «plus» dans les publicités pour de tels mariages: «Pack mariage tout compris, la mariée est livrée chez le mari au bout de trois mois, séjour pour mariage au Viêt Nam durant seulement six jours (pour éviter toute velléité de fuite, note de l'auteur). Quatre garanties: virginité, livraison en trois mois, pas d'augmentation du tarif, si la mariée s'enfuit, remplacement assuré par une autre mariée. Contacter... (suit un numéro de téléphone)». Ou bien: «Mariée vietnamienne à 20000 yuan, garantie vierge, pas de fuite». Voir aussi Trafficking In Persons, June 2007, p. 17 : «Vietnamese - They Don't Run Away!». http://www.state.gov/g/tip/rls/tiprpt/2007/. Selon le Liberty Times (Taiwan, 30/07/2005), plus de 12000 mariages arrangés entre Taiwanais et Vietnamiennes ont été contractés en 2004, alors qu'il n'y en avait qu'environ 3800 avec des Américains dont bon nombre sont d'origine vietnamienne. Voir aussi : Nicolas Lainez, «Transactions de personnes et formes de dépendance au Vietnam. Pour une anthropologie de la "Traite des Jaunes" ». Mémoire de Master 2 en anthropologie sociale et ethnologie. Paris, EHESS, 2007. L'auteur rédige actuellement une thèse sur la prostitution au Viêt Nam. 
ample informé, peu, ou pas du tout de fausses vierges autochtones, dans les pays riches, «États de droit» par surcroît, comme au Japon, voire en Corée (du Sud). En revanche, ces «denrées frelatées», pour reprendre une expression de l'écrivain Yu Hua, en Chine, d'origine autochtone, mais aussi ${ }^{76} \mathrm{~d}^{\text {' } ~}$ importation», sont présentes à Taiwan, à Singapour, aux Philippines, en Thaïlande, au Laos, au Cambodge ${ }^{77}$... Bref, la falsification de la virginité est presque une industrie intra-asiatique, elle s'adresse en priorité à une clientèle de gens nantis. Les rapports sexuels demeurent ce qu'ils ont souvent été, inégalitaires, mais cette fois ils n'entrent plus dans ce cadre des relations «Nord-Sud» classiques.

Telles sont nos premières conclusions. Notre étude souffre encore tout autant de lacunes documentaires et statistiques que d'insuffisances dans les enquêtes sur le terrain. Que le lecteur veuille bien considérer ce texte comme les premières pages, à peine esquissées, d'un travail qui reste à faire. Nous avons souvent recours aux témoignages littéraires, faudrait-il y voir un hommage implicite aux écrivains, souvent plus diserts sur ces phénomènes de société, et certainement plus sensibles à cette dimension commerciale du corps? Néanmoins, une question demeure, sans véritable réponse: qu'est ce qui pousse des Asiatiques à vouloir jouir de la souffrance d'autres Asiatiques, lesquelles, pour pouvoir leur offrir ce qu'elles n'ont plus, doivent subir des modifications de la partie la plus intime de leur corps? Sont-ils dans l'ignorance que de telles pratiques existent? Ils ne doivent plus l'être, à présent. Leur quête du plaisir hédonistique est elle si grande qu'elle dépasse toute autre valeur, non pas seulement morale, religieuse, mais simplement humaine? Jusqu'où peut aller notre aliénation qui fait de l'aliénation d'autrui la source de notre jouissance? Sommes-nous dans des sociétés, proches certes, mais où d'anciennes valeurs communes ont totalement disparu? Chercheur impliqué, pourquoi esquiverions-nous ce questionnement?

76. Sinon «surtout», pour des raisons de déséquilibre démographique déjà évoquées (voir: En espérant un fils, op. cit., p. 97-98. L'enquête intercensitaire de 2005 donne 120,5 naissances de garçons pour 100 naissances de filles, en Chine. Le phénomène est commun à d'autres pays d'Asie: Inde, Corée du Sud, Taiwan...).

77. Nous connaissons mal la situation dans les pays asiatiques et musulmans. 